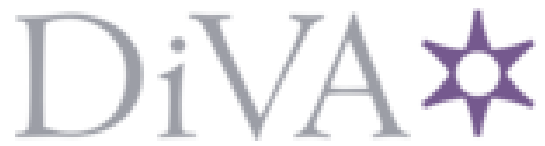

http://www.diva-portal.org

Preprint

This is the submitted version of a paper presented at Acoustofluidics 2019 25-28 August 2019 Enschede, Netherlands.

Citation for the original published paper:

Werr, G., Khaji, Z., Ohlin, M., Andersson, M., Klintberg, L. et al. (2019)

Integrated thin film resistive sensors for in situ temperature measurements in an acoustic trap

In: Acoustofluidics 2019: This annual meeting will be held in Twente, The Netherlands in 2019. This focused meeting is dedicated to exploring the science, engineering, and use of micro- to nanoscale acoustofluidics. (pp. 140-141).

N.B. When citing this work, cite the original published paper.

Permanent link to this version:

http://urn.kb.se/resolve?urn=urn:nbn:se:uu:diva-398685 


\title{
Integrated thin film resistive sensors for in situ temperature measurements in an acoustic trap
}

\author{
Gabriel Werr ${ }^{1}$, Zahra Khaji ${ }^{1}$, Mathias Ohlin ${ }^{1,2}$, Martin Andersson ${ }^{1}$, Lena Klintberg ${ }^{1}$, \\ Sean S. Searle ${ }^{1,3}$, Klas Hjort ${ }^{1}$, and Maria Tenje ${ }^{1,4}$ \\ ${ }^{1}$ Department of Engineering Sciences, Uppsala University, 72129 Uppsala, Sweden \\ ${ }^{2}$ Now at Aimpoint AB, 21375 Malmö, Sweden \\ ${ }^{3}$ Department of Biomedical Engineering, National University of Singapore, Singapore 117583, Singapore \\ ${ }^{4}$ Science for Life Laboratory, Sweden \\ E-Mail: maria.tenje@ angstrom.uu.se URL: https://www.teknik.uu.se/mikrosystemteknik/embla/
}

\begin{abstract}
Introduction
Microfluidic devices offer a precise and rapid assay platform with fully controlled microenvironment conditions with regards to $\mathrm{pH}$ and chemical composition. Some assays require particle manipulation for sorting, incubation or perfusion and here acoustophoresis offers a non-contact and label-free method to achieve this. Acoustic trapping is a method to retain microparticles in a microfluidic channel against a flow and its use has already been demonstrated for sample enrichment [1], and multistep bead-based assays [2]. One challenge when using acoustic trapping for biological samples is the heating effects from the piezoelectric transducers (PZT) used to actuate the system. The common method to control and measure the temperature is by measuring the external temperature of the PZT in the assumption the internal temperatures of the chip will be lower than that of the heat source [3]. Here, we present an acoustic trap with integrated temperature sensors, measuring the in situ temperature of the trap comparing it to five external measuring points. With the system we were able to measure temperature variations in the range of $\pm 0.01{ }^{\circ} \mathrm{C}$. Our goal was to test the hypothesis, whether the PZT would always be the hottest position during operation. Our results confirmed this assumption for the system without external cooling, but refuted it with applied external convective cooling. This demonstrates the need for integrated temperature sensors to monitor all microenvironmental aspects in an acoustic trap.
\end{abstract}

\section{Methods}

Figure 1 shows the design of the acoustic trap with two integrated thin film resistive sensors (RTD). The chip consists of two glass layers, where the channel and the trap were etched in the bottom glass, while the RTDs were sputtered onto the top glass. The top glass was then bonded to the bottom glass with RTD1 positioned to form the ceiling of the trap and RTD2 placed underneath the external PZT. The PZT was glued on top of the glass chip and the fluidic connection was achieved by gluing a capillary sheathed with metal tubing to the chip [4], [5].

For the temperature measurements the RTDs and a Thermocouple (TC2), to compensate for room temperature variations, were calibrated to a reference Thermocouple (TC1), which was placed on the five external measurement positions. The trap was tested for influences by different bead sizes, conductance of the liquid, and cell suspensions, with no negative effects observed. For the main temperature measurements DI-water was used. The measurement cycles consisted of $20 \mathrm{~min}$ with the PZT actuated followed by $20 \mathrm{~min}$ where the PZT was switched off. For each position the cycle was repeated $n=20$ times at stop-flow with the PZT at $20 \mathrm{~V}_{\mathrm{pp}}$ and $1.83 \mathrm{MHz}$. Additionally, a parametric study was conducted with flow rates from $0.2 \mu \mathrm{l} / \mathrm{min}$ to $20 \mu \mathrm{l} / \mathrm{min}$ and PZT voltages from $10 \mathrm{~V}_{\mathrm{pp}}$ to $20 \mathrm{~V}_{\mathrm{pp}}$, with $\mathrm{n}=5$.
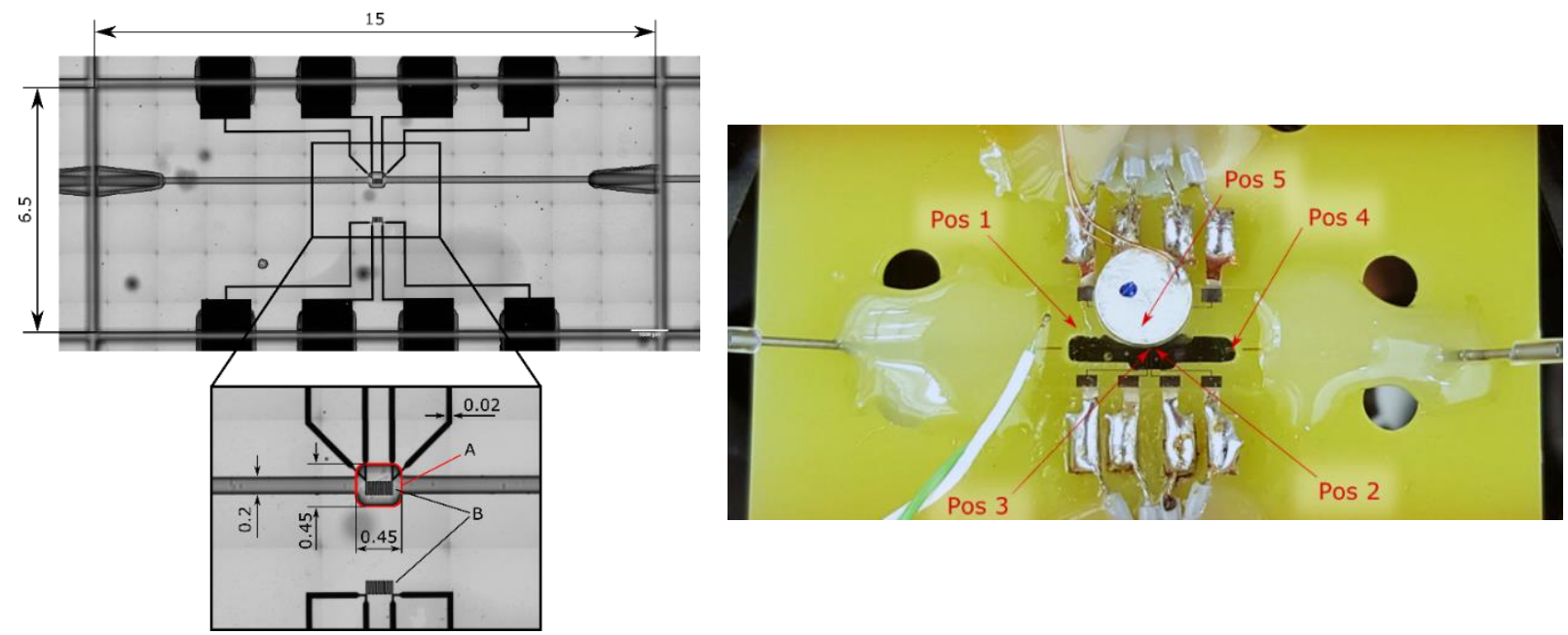

Figure 1: To the left the design of the acoustic trap with integrated thin film sensors and the relevant dimensions. To the right the assembled system with connected tubing, sensors and piezo, and the five external measurement spots. 
The results were pooled to calculate average values and standard uncertainties at a confidence level of $95 \%$.

\section{Results}

The integrated RTDs performed without disturbance both when beads and cells were trapped. We observed no
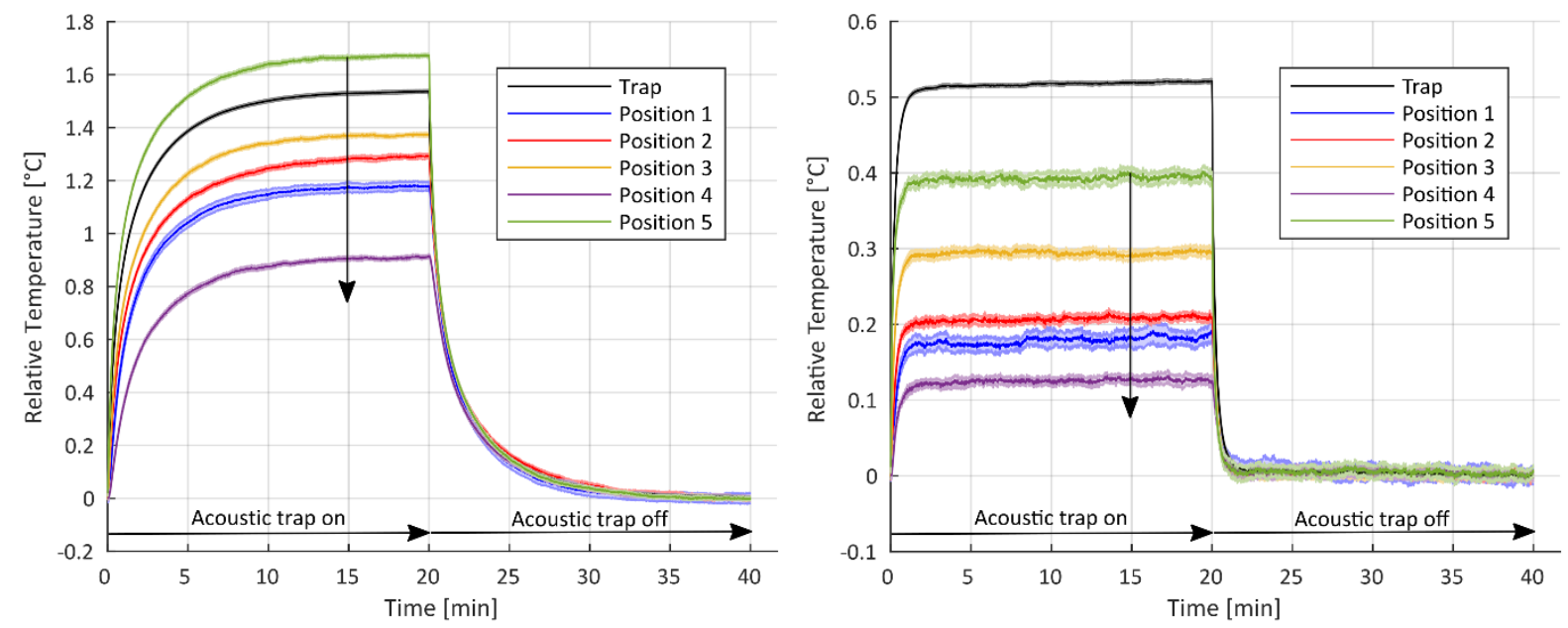

Figure 2: External temperature of the chip relative to room temperature at five different positions with standard uncertainty $(n=20)$. Black line shows internal trap temperatures of RTD1 $(n=100)$. The arrow indicates the distance of the sensor to the trap. In the left graph temperatures without external cooling are shown, while the right graph shows temperatures with external cooling. A change in the order can be seen for Position 5 and Trap

differences in the measured temperatures when using DI water or conducting liquids. Upon actuation, the temperature increased at all measurement positions. With no external cooling applied, Position 5 (on top of the PZT) measuring the highest temperature increase of $1.67 \pm 0.01{ }^{\circ} \mathrm{C}$. When external convective cooling was applied on the chip the hottest reported temperature was observed inside the trap, at $0.52 \pm 0.01{ }^{\circ} \mathrm{C}$. Position 5 , only reported an increase of $0.4 \pm 0.01^{\circ} \mathrm{C}$. The same trends were observed with fluid flow in the channel.

\section{Conclusion}

We present an acoustic trap with integrated thin film sensors, that for the first time allows in situ temperature measurements during operation. From our results we find the temperature increase inside the acoustic trap to be a result of the dissipative actuation of the PZT. The most important finding is that the temperature inside the acoustic trap can be higher than the temperature measured directly on top of the PZT.

\section{References}

[1] J. V. Norris, M. Evander, K. M. Horsman-Hall, J. Nilsson, T. Laurell, and J. P. Landers, “Acoustic Differential Extraction for Forensic Analysis of Sexual Assault Evidence," Anal. Chem., vol. 81, no. 15, pp. 6089-6095, Aug. 2009.

[2] M. Tenje et al., "Acoustic trapping as a generic non-contact incubation site for multiplex bead-based assays," Anal. Chim. Acta, vol. 853, no. 1, pp. 682-688, 2015.

[3] P. Augustsson, R. Barnkob, S. T. Wereley, H. Bruus, and T. Laurell, "Automated and temperature-controlled micro-PIV measurements enabling long-term-stable microchannel acoustophoresis characterization," Lab Chip, vol. 11, no. 24, pp. 4152-4164, 2011.

[4] M. Andersson et al., "Thin film metal sensors in fusion bonded glass chips for high-pressure microfluidics," $J$. Micromechanics Microengineering, vol. 27, no. 1, 2017.

[5] M. Ohlin, I. Iranmanesh, A. E. Christakou, and M. Wiklund, "Temperature-controlled MPa-pressure ultrasonic cell manipulation in a microfluidic chip," Lab Chip, vol. 15, no. 16, pp. 3341-3349, 2015. 